\title{
Estudio Experimental para la Predicción del Funcionamiento de Evaporadores
}

Luis A. Rubio, Raúl E. Chernikoff, Ernesto Muñoz y Sergio A. Sini

Facultad de Ciencias Aplicadas a la Industria (U.N.Cuyo)

San Martín 358, San Rafael, Mendoza-Argentina. (e-mail: larubio@fcai.uncu.edu.ar)

\section{Resumen}

Se diseñó y construyó un evaporador a escala industrial de un tubo, que puede ser armado según seis principios diferentes. Se desarrolló un modelo basado en la identificación y reducción de variables y se realizó una evaluación experimental operando el evaporador en circulación forzada ascendente. Los datos se relacionaron aplicando el modelo global y se obtuvo una ecuación predictiva simple en función de variables macroscópicas independientes. Posteriores investigaciones han de conducir a la reconciliación de la ecuación obtenida para el modelo global con aquellas a obtener por aplicación del modelo local.

Palabras clave: evaporación, modelo matemático, circulación forzada, diseño

\section{Experimental study to predict evaporators performance}

\begin{abstract}
In this work, an industrial scale evaporator with one tube, that can be assembled in six different ways, was designed and constructed. A model based on the identification and reduction of variables was developed and an experimental evaluation was made by operating the evaporator in a climbing forced circulation. The data were related applying the global model and a simple predicting equation as function of independent macroscopic variables was obtained. Further research should include reconciliation of the equation obtained for the global model with those to be obtained by the application of the local model.
\end{abstract}

Keywords: evaporator, mathematical model, forced circulation, design 


\section{INTRODUCCIÓN}

Los estudios sobre evaporadores existentes en la literatura, ya sea a escala piloto o industrial, han sido realizados sobre equipos distintos unos de otros de configuración fija en los que se asume que los resultados obtenidos son válidos a una escala distinta y por ello también comparables por el principio de similitud.

Sin embargo, en fenómenos que implican simultáneamente transferencias de masa, calor y cantidad de movimiento, como es el caso de la evaporación, la similitud sólo puede lograrse en forma parcial. Esto limita seriamente la validez de las ecuaciones actualmente en uso que se basan, todas ellas, en el marco teórico que postula la existencia de mecanismos aditivos de transmisión de calor micro y macroconvectivos (Chen, 1966) y ha llevado a cuestionar los resultados obtenidos para este tipo de fenómenos (Cao, 1995). Por otro lado, una revisión crítica de los distintos métodos modelísticos (Rubio, 2003) ha constatado las inconsistencias del marco teórico en uso en el fenómeno de evaporación, que se observa aún en trabajos recientes (Kamil-Alam, 1996), (Schneider, 2005), (Cheng et al, 2006).

En el camino de formular un nuevo enfoque sobre los fenómenos de transferencia complejos, se han logrado resultados parciales con respaldo experimental.

\section{OBJETIVOS}

Para el presente trabajo se propone:

- Construir un evaporador que permita la realización de estudios comparativos de los distintos principios de evaporación.

- Proponer un método para la selección de variables macroscópicas significativas.

- Evaluar experimentalmente el equipo en una de sus configuraciones.

\section{METODOLOGÍA Y RESULTADOS}

El trabajo se plantea en tres niveles, a saber:

\section{Diseño}

De un evaporador que pueda ser configurado de diferentes maneras, cuyo tubo de intercambio sea de las dimensiones de los de uso industrial (Chernikoff, 2003).
Para ello, la estructura del evaporador se divide en:

Estructura no configurable: donde se agrupan los elementos comunes a todos los tipos de evaporadores, mostrada en la Figura 1.

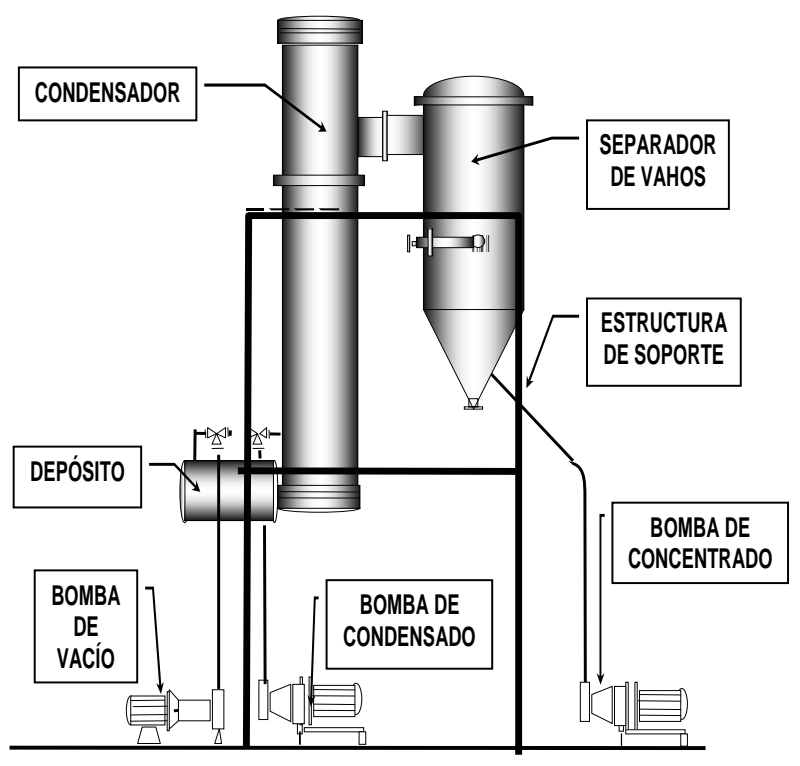

Fig. 1: Estructura no configurable, de posición fija

Estructura configurable: Incluye partes como intercambiadores de calor, bomba de recirculación y cañerías de conexión que al ser dispuestas de un modo dado permiten obtener una configuración determinada del evaporador, mostrada en la Figura 2.

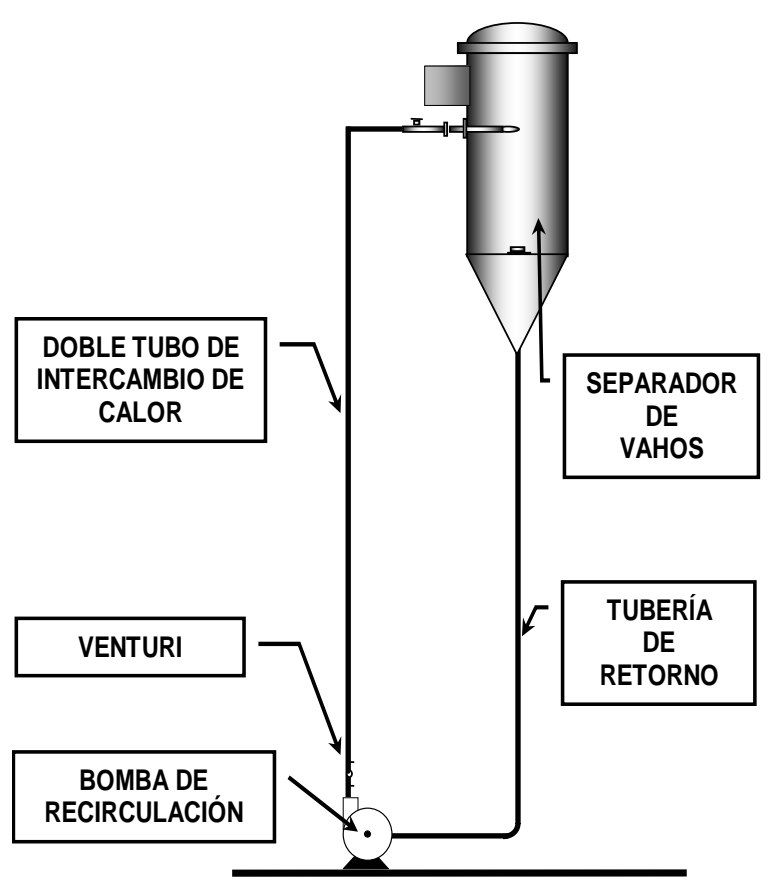

Fig. 2: Estructura configurable dispuesta como evaporador de circulación forzada ascendente

Información Tecnológica - Vol. 18 No 2 - 2007 
Las distintas configuraciones que el equipo diseñado admite son: evaporador de film descendente; evaporador de film ascendentedescendente; evaporador de film ascendente en tubo largo vertical, tipo Kestner; evaporador de circulación forzada sin ebullición dentro de tubo, horizontal con columna hidrostática en descarga; evaporador de circulación forzada con ebullición dentro de tubo operando en el sentido vertical-descendente opuesto al flujo natural de los vahos, tipo Rossi \& Catelli; evaporador de circulación forzada con ebullición dentro de tubo operando en el sentido vertical-ascendente del flujo natural de los vahos.

\section{Identificación y selección de variables macroscópicas}

Las variables macroscópicas identificadas en la bibliografía se resumen en la Tabla 1.

Tabla 1: Resumen de variables macroscópicas

\begin{tabular}{|c|c|}
\hline $\begin{array}{l}\text { Primer grupo: } \\
\text { Parámetros } \\
\text { geométricos } \\
\text { y } \\
\text { constructivos }\end{array}$ & $\begin{array}{l}\text { 1. Tipo de configuración } \\
\text { 2. Diámetro del tubo (d) } \\
\text { 3. Largo del tubo (L) } \\
\text { 4. Material de construcción } \\
\text { 5. Espesor de pared del tubo } \\
\quad\left(e_{w}\right) \\
\text { 6. Naturaleza de la } \\
\left.\text { superficie ( } r_{w}\right)\end{array}$ \\
\hline $\begin{array}{l}\text { Segundo } \\
\text { Grupo: } \\
\text { Parámetros } \\
\text { operativos: }\end{array}$ & $\begin{array}{l}\text { 7. Velocidad media de } \\
\text { producto }(\mathrm{u}) \\
\text { 8. Temperatura de vahos } \\
\left(\mathrm{T}_{\mathrm{V}}\right) \\
\text { 9. Temperatura de vapor de } \\
\text { calefacción }\left(\mathrm{T}_{\mathrm{S}}\right) \\
\text { 10. Diferencia de } \\
\text { temperatura } \\
\text { 11. Alejamiento de la } \\
\text { temperatura de equilibrio } \\
\text { 12. Concentración de sólidos } \\
\text { solubles }\left(\mathrm{c}_{\mathrm{C}}\right) \\
\text { 13. Tipo de producto }\end{array}$ \\
\hline $\begin{array}{l}\text { Tercer } \\
\text { grupo: } \\
\text { Propiedades } \\
\text { del fluido } \\
\text { líquido a la } \\
\text { temperatura } \\
\text { de la masa } \\
\text { del producto }\end{array}$ & $\begin{array}{l}\text { 14. Temperatura de la masa } \\
\text { de producto }\left(\mathrm{T}_{\mathrm{i}}\right) \\
\text { 15. Tensión superficial }(\sigma) \\
\text { 16. Viscosidad: }\left(\mu_{\mathrm{L}}\right) \\
\text { 17. Calor específico } \\
\text { 18. Conductividad térmica } \\
\text { 19. Calor latente de } \\
\text { vaporización } \\
\text { 20.Gradiente crítico de } \\
\text { temperaturas ( } \Delta \text { Tcrítico) } \\
\text { 21. Elevación del punto de } \\
\text { ebullición }\end{array}$ \\
\hline
\end{tabular}

Las variables antes mencionadas pueden ser reducidas de la siguiente manera:

Considerando el modelo local como una caja negra, se reducen las propiedades del tercer grupo a dos parámetros operativos, por aplicación de la regla de las fases: temperatura de producto, temperatura exterior y concentración de sólidos solubles.

Considerando el modelo global como una caja negra, y a partir de la definición de variable independiente, se obtienen como variables macroscópicas: tipo de producto; temperatura de vapor de calefacción $\left(T_{S}\right)$; temperatura de vahos $\left(T_{v}\right)$; velocidad media de producto $(u) ; y$ concentración de sólidos solubles $\left(\mathrm{c}_{\mathrm{C}}\right)$

Pudiéndose, además reducir los parámetros de temperatura del modelo local a los ya elegidos para el modelo global.

Matemáticamente el modelo global ha de expresarse:

$q=f\left(T_{s}, T_{v}, u, c_{c}\right)$

\section{Evaluación experimental}

Se realizaron doce ensayos con agua y soluciones de sacarosa, en los que se midieron los parámetros operativos para doce condiciones distintas de funcionamiento, las que se determinaron por diseño factorial fraccionario. Para cada condición de operación se tomaron 150 lecturas de cada variable. Los datos obtenidos utilizando el sistema de adquisición TAS $^{\circledR}$, Taurus Active Scada, fueron volcados a una planilla electrónica EXCEL ${ }^{\circledR}$ donde se equiespaciaron y se recortaron aquellos correspondientes a los estados estacionarios. Se calcularon la media y la desviación standard. Con tales datos se realizaron los cálculos necesarios para los balances.

Finalmente, utilizando el programa comercial STATGRAPHICS PLUS $4.0^{\circledR}$ se realizó una regresión múltiple de donde se obtuvo la ecuación predictiva.

Se trabajó sobre la base de una expresión potencial de la forma:

$y=b_{0} \cdot x_{1}^{b 1} \cdot x_{2}^{b 2} \cdots x_{n}^{b n}$ 
A continuación se muestra la ecuación propuesta (ec. 3) y sus estadígrafos en la Tabla 2.

$\mathrm{q}=4,184 .\left(\frac{\mathrm{T}_{\mathrm{V}}^{1 / 4} \cdot c_{C}^{4}+12 \cdot u^{2}}{T_{V} \cdot u^{2}}\right)^{0,58} \cdot\left(T_{S}-T_{V}\right)$

Tabla 2: Estadígrafos del análisis de regresión

\begin{tabular}{|c|c|c|c|}
\hline \multicolumn{4}{|c|}{ Variable dependiente: In $\mathrm{q}$} \\
\hline parámetro & estimado & $\begin{array}{c}\text { error } \\
\text { estándar }\end{array}$ & valor $\mathrm{P}$ \\
\hline $\ln \mathrm{T}_{\mathrm{v}}{ }^{0.25} \cdot \mathrm{c}_{\mathrm{C}}{ }^{4}+12 \mathrm{u}^{2}$ & 0,580 & 0,228 & 0,0134 \\
\hline $\ln \left(\mathrm{T}_{\mathrm{v}} \cdot \mathrm{u}^{2}\right)$ & $-0,581$ & 0,168 & 0,0009 \\
\hline $\ln (\Delta \mathrm{T})$ & 1,012 & 0,117 & 0,0000 \\
\hline $\mathrm{R}^{2}$ ajustado: 96,8 \\
\hline Durbin - Watson: 1,776 \\
\hline
\end{tabular}

\section{CONCLUSIONES}

Se diseñó y construyó un evaporador para investigación y docencia que puede ser configurado de seis formas distintas, a fin de realizar estudios comparativos entre estas configuraciones y obtener resultados a escala industrial.

El sistema de adquisición de datos permitió trabajar con 150 lecturas para cada uno de los datos medidos en estado estacionario. Las corridas fueron repetidas además dos veces, de acuerdo con un diseño estadístico previo.

Se desarrolló un método modelístico basado en los conceptos de modelo de caja negra y regla de las fases para la obtención de una ecuación predictiva en función de variables independientes macroscópicas.

A partir de los datos recogidos en el modo de circulación forzada ascendente en estado estacionario se preparó un modelo sencillo en función de variables macroscópicas que da cuenta, en gran medida, de la variabilidad de la información experimental obtenida.

Lo realizado hasta el presente sólo es la primera parte de un trabajo a continuar con la reconciliación de la ecuación hallada para el modelo global con las distintas ecuaciones que se han de obtener del modelo local.

\section{REFERENCIAS}

Cao, E., "Correlaciones para diseño de reboilers del tipo kettle", Procesos, Marzo, 1018, (1995).

Chen, J. C., "Correlation for boiling heat transfer to saturated fluids in convective flow", Ind. Eng. Chemistry Proc. Des. Dev., 5 (3), 322-329 (1966).

Cheng, L. ; Ribatski G.; Wojtan, L.; Thome, J. "New flow boiling heat transfer model and flow pattern map for carbon dioxide evaporating inside horizontal tubes", International Journal of Heat and Mass Transfer, Vo 49, Is., 40824094, 21-22, October 2006.

Chernikoff, R., "Diseño, construcción y evaluación experimental de un evaporador tubular en su configuración de circulación forzada", Tesis doctoral, Universidad Politécnica de Valencia, Valencia-España, (2003).

Kamil, M.- Alam, S. "Experimental study of heat transfer to boiling binaries in a reboiler tube" Int. J.Heat Mass Transfer. Vol. 39, № 7, 1489-1501 (1996).

Rubio, L. y otros 6 autores, "Una nueva mirada a los fenómenos de transferencia: aplicación al caso de evaporadores", Memorias del IV Congreso Iberoamericano de Ingeniería de Alimentos (CIBIA IV), Viña del Mar (Chile), Septiembre, 30 (2003)

Rubio, L., "Técnica de medición en estado cuasi-estacionario aplicada a evaporadores", Tesis doctoral, Universidad Politécnica de Valencia, Valencia-España, (2003).

Schneider, K. "Adaptative modelling: an intuitive approach for the applicattion of irreversible thermodynamics to multiphase flow simulator". Nuclear Engineering and Design 235 2514- 2526 (2005). 